\title{
ZEITSCHRIFT
}

\section{FÜR KUNSTGESCHICHTE}

Begründet von Wilhelm Waetzoldt und Ernst Gall

Herausgegeben von Margarete Kühn

unter Mitwirkung von Georg Kauffmann

Cornelius Müller Hofstede und Hans Thümmler 
Gedrudkt mit Unterstützung der Deutschen Forschungsgemeinschaft. Deutscher Kunstverlag G.m.b.H., München-Berlin Drudk: Mittelbayerische Druckerei- und Verlagsgesellschaft mbH., Regensburg 


\section{Helldunkel und Zeitlichkeit Caravaggio, Ribera, Zurbaran, G. de La Tour, Rembrandt}

Problematik der Zeit ist bei geistesgeschichtlicher Betrachtung des Barockzeitalters in den letzten Jahrzehnten immer stärker in den Blickpunkt getreten. Selbst in der Kunstgeschichte ist man auf das Problem aufmerksam geworden, dabei scheint das Medium bildende Kunst zunächst der Zeit verschlossen zu sein, da es sich nicht im Zeitablauf vollzieht. Bildende Kunst bewegt sich nicht, kinetische Kunst ist erst heute mehr als eine Randerscheinung. Im folgenden soll anhand einer malerischen Technik, die typisch für die Barockmalerei ist, dem Helldunkel, die Bedeutung der Zeitlichkeit aufgezeigt werden. Ein solcher Versuch ist in dieser Weise bis jetzt noch nicht unternommen worden. Über die bereits vorhandenen Vorarbeiten wird am Ende des Aufsatzes referiert, ebenfalls finden sich dort noch einige Bemerkungen zur hier angewandten Methode der philosophischen Interpretation.

\section{Caravaggio}

Die Bedeutung des Helldunkels, die seit dem späten Mittelalter immer größer wird, erreicht bei Caravaggio einen Höhepunkt. Doch schon bei Leon Battista Alberti und Leonardo da Vinci nehmen die Probleme des Lichts eine zentrale Stelle in den theoretischen Schriften ein. Grundlegend ist für ihre Betrachtung die Unterscheidung zwischen Lichtquelle einerseits und Licht auf dem Gegenstand andrerseits (Luce und Lumen bei Leonardo); die Beziehung und gegenseitige Abhängigkeit von Licht und Schatten werden genau untersucht. Der Theorie entspricht die Darstellungsart des Lichts in der gleichzeitigen Malerei. Die Dinge werden als beleuchtete gezeigt, mit Licht- und Schattenseite. Die hochmittelalterliche Malerei war schattenlos; sie kannte noch nicht den Unterschied zwischen Licht, das erhellt, und beleuchteter Materie. Das Dargestellte besaß sein Eigenlicht ${ }^{\mathrm{I}}$. Mit der Einführung der Beleuchtung entsteht ein Unterschied zwischen dem ungegenständlichen Phänomen Licht und der Gegenständlichkeit. Die Erscheinungsweise der Dinge wird abhängig vom Licht, Erscheinungsweise und Wesen des Dinges fallen auseinander. Es entsteht eine Kunst des Scheins ${ }^{2}$. Durch diese Bedingtheit ist das Dargestellte nicht etwas, was immer so aussieht, sondern etwas, was unter bestimmten Voraussetzungen, zu einem bestimmten Zeitpunkt so sich zeigt. Das Dargestellte ist damit verzeitlicht. Würde sich die Beleuchtung ändern, stellte sich das Gezeigte anders dar, - und die Möglichkeit von Änderung gibt es nur innerhalb der Kategorie Zeit. Ein paralleles Phänomen ist die gleichzeitig aufkommende Zentralperspektive, die ebenfalls die Bedingtheit der Anschauung zum Ausdruck bringt. Auch die Beleuchtung selbst hat eine räumliche Komponente: denn Licht- und Schattenverteilung hängen ab von der Stellung des Gegenstandes im Raum, das Licht kommt aus bestimmter Richtung. Raum und Zeit zeigen sich als abhängig voneinander, Zeit ermöglicht Veränderung im Raum (und damit Erfahrung von Raum). Beides, Zeit und Raum, sind Voraussetzungen für das Helldunkel, in ihm sind sie enthalten. Die vom Helldunkel betroffenen Gegenstände sind nicht mehr in der raum- und zeitlosen Ewigkeit Gottes aufgehoben, wie man die Erscheinungsweise hochmittelalterlicher Malerei beschreiben könnte. Das Helldunkel ist eine Säkularisationserscheinung. Das Dargestellte zeigt sich in irdischer Bedingtheit. Eine Polarität Sein - Schein, immer - jetzt, 
gültig - zufällig bricht auf. Freilich werden diese Antinomien in der Renaissancemalerei nicht betont. Die malerische Säkularisation führte eher zu einer befreiten Hinwendung zum Irdischen. Leonardo empfiehlt immer wieder, man möge für die Malerei diffuses Licht wählen (also zum Beispiel bedeckten Himmel); solche Beleuchtung bringt die Dinge am besten zur Darstellung. Sie ist Mittelwert und als solcher wird sie nicht aufdringlich. Die Schatten sind nur schwach, gerade so, daß die Modellierung der Körperlichkeit zum Ausdruck kommt, doch ohne daß durch das Licht die Einheit der Gegenstände zerrissen würde und die Beleuchtung die Form der Dinge überspielte. Die Beleuchtung kommt selbst also kaum thematisch dem Beschauer zu Bewußtsein, sie ist der Darstellung der Dinge durchaus dienstbar. Insofern kann mit Wölfflin immer noch von einer Kunst des Seins die Rede sein, auch wenn dieses Sein nicht mehr unbedingt ein Ewiges ist. -

Bei Caravaggio allerdings ist dann die Bedeutung des Helldunkels eine andere. In seiner mittleren Zeit sucht er extreme Helldunkelgegensätze. Damit erscheint das Dargestellte nur noch fragmentarisch. Einzelne Teile, oft durchaus nebensächliche, leuchten grell auf, anderes verschwindet im Dunkel. Nur was vom Licht getroffen wird, kann erscheinen. Leonardos Erkenntnisse werden auf die Spitze getrieben, das Luce scheint willkürlich mit der Erscheinungswelt zu verfahren. Schlaglichtartig fällt es in den dunklen Bildraum, seine Quelle wird nicht gezeigt: so wirkt es als absolute Bedingung für das Erscheinen der Dinge. Ohne Licht wäre nichts da, wäre Finsternis. Noch nie ist Dunkelheit als Nichts, als Negation, als Tod in der Malerei so präsent gewesen. Die Bedingtheit der Erscheinungswelt ist zum bedrängenden Problem geworden, Vergänglichkeit zur dominierenden Macht.

Die Herrschaft der Zeit drückt sich im Augenblickscharakter von Caravaggios Bildern aus. Wie ein Scheinwerferstrahl, der weggeblendet werden könnte, fällt das Licht in den Bildraum. Es kommt nicht nur von einem unbekannten Außen, es bleibt auch dem Dargestellten fremd. Es prallt auf der Oberfläche der Dinge ab. Caravaggios Farbauftrag hat etwas Aufpoliertes, die Oberfläche der Dinge ist Grenze, so daß das Licht überhaupt nicht mit den Dingen sich zu verbinden scheint. Der Eindruck eines Materie-Farbe-Licht-Gemischs wie etwa in der venezianischen Malerei des 16. Jahrhunderts entsteht in keiner Weise. Den Farben scheint das Licht als bloße Ursache ihrer Erscheinung oder ihres Verschwindens fremd zu sein, es entsteht der Eindrudk einer Feindschaft zwischen Farbe und Licht. Diese Fremdheit des Lichts im Bildraum wird noch durch seine Kälte unterstrichen, durch die es wie reine physikalische Kategorie wirkt. Der heftige Einfall verbunden mit der erstarrten drastischen Gestik der Personen erweckt den Eindruck der Plötzlichkeit. Das Immersein der mittelalterlichen Malerei ist bei Caravaggio zur irdischen Augenblicklichkeit geworden. Das ist eine äußerste Form der Vergänglichkeit. So leben diese Gemälde von einem seltsamen Gegensatz: In der Augenblicklichkeit ist die Zeit thematisch, aber im Festhalten des Augenblicks (man hat von Momentaufnahmen gesprochen) ist die Zeit zugleich aufgehoben. Dieses Aufheben ist nicht eine Vereinigung von Augenblick und Zeit zur Dauer. Es ist gebannter Augenblick; das Bewußtsein, daß es nur ein Augenblick ist, bleibt bestehen. So steht das Gemälde im Widerspruch zu sich selbst. Es verleiht dem Dauer, was keine Dauer hat. Das ist das Provokante von Caravaggios Helldunkel. Seine Malerei war nicht bloß eine Herausforderung seiner Zeit, weil sie die bisherige Malweise revolutionierte, vielmehr trägt sie den Charakter der Provokation als ungelösten Widerspruch in sich selbst. Darum empfindet der Betrachter eine ungelöste Spannung. Die emotionale Wirkung solcher Malerei hängt mit dieser Zeitlichkeit zusammen. Diese Bilder zeigen als fixierter Augenblick nicht Immerseiendes, geben dem Betrachter nicht etwas zu dauerndem Besitz (wie es zum Beispiel das klassische Verständnis 


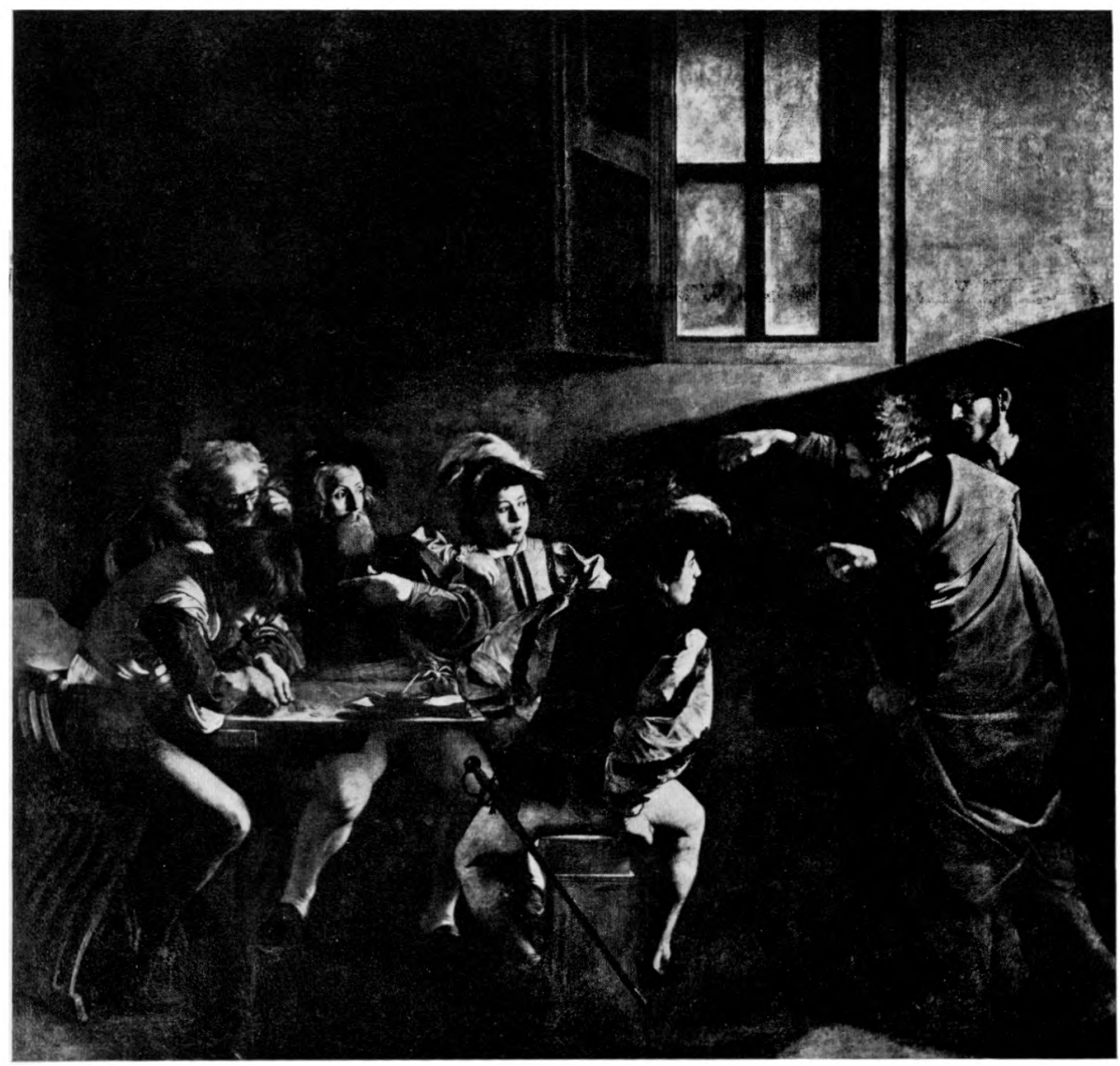

I. Caravaggio, Berufung des Matthäus. Rom, S. Luigi dei Francesi

von Schönheit will). Vielmehr bewirken sie Emotionen, die ein durchaus zeitliches Phänomen sind. Emotion ist Veränderung, Bewegung des Gefühls. (Man vergleiche dazu, wie Dichtung, die in erster Linie Emotionen wecken will, meistens mit stark dynamisiertem, insofern zeithaltigem, Wortschatz arbeitet, gefühlsbestimmte Musik mit Crescendo und Decrescendo, diesen typischen Zeitphänomenen, die dem Helldunkel verwandt sind.) Der Betrachter wird also selbst in einen zeitlichen Vorgang hineingerissen. Daß die Zeitlichkeit im Bild zugleich fixiert ist, die Emotion nicht durch das Bild weitergeführt wird (vgl. die anderen Möglichkeiten der zeitgebundenen Künste Musik und Dichtung), schafft einen Widerspruch im Gefühl, der ungelöst bleibt. Das Helldunkel, das in seiner Schärfe die Zeit als Augenblicklichkeit bewußt macht, das als Einfallendes zeitlichen Geschehenscharąkter hat, schafft als Fixiertes zugleich Spannung in und durch sich selbst. Es sei nur nebenbei erwähnt, daß der Realismus Caravaggios zu einem ähnlichen Widerspruch führt. Denn Realistisches hat in sich auch den Charakter des jetzt gerade so Seienden (des nicht überzeitlich Typischen). Dieses Zufällige ist im Bild aber herausgehoben und fixiert, soll bei religiöser Thematik sogar etwas von überzeitlicher Gültigkeit sein. Der zeitgenössische Protest gegen Caravaggios Realismus entsprang also nicht nur der Konfrontation zweier Ansichten, sondern hatte seine Begründung durchaus in einer Widersprüchlichkeit in 
Caravaggios Malerei selbst. Mit solcher Widersprüchlichkeit waren den Caravaggionachfolgern von der Sache her Probleme gestellt. Die Barockmalerei ist in vielem Auseinandersetzung mit dem Phänomen Caravaggio, und Caravaggio selbst hat in seinen letzten Bildern neue Wege der Helldunkelmalerei gesucht. Zusammen mit einer Milderung der Plötzlichkeit des Lichteinfalls schafft er eine atmosphärische Erfülltheit des Raums. Das Nächtige ist so nicht mehr bloß drohender Hintergrund, ein Nichts: es wird zum tragenden Grund.

Entschiedener in neuer Richtung geht Ribera, der nach Caravaggio in Neapel zum führenden Maler wurde.

\section{Rib e r a}

Der Lichteinfall ist in Riberas Bildern weniger heftig, ihm fehlt der ausgesprochene Charakter des Plötzlichen. Man meint nicht, das Licht könnte jederzeit weggeblendet werden. Die Oberfläche des Dargestellten ist nicht glatt, vielmehr verfängt sich das Licht in ihr, so daß ein reiches Farb-, Ton- und Lichtspiel entsteht. So vielfältig wie bei Ribera sind vorher noch nie Glanzund Splitterlichter, Reflexe und Abschattierungen verwendet worden. Caravaggios Antagonismus zwischen Licht und Materie ist damit gemildert, durch die Vermittlung wird ein überreiches Spiel an Tonabschattungen und Farbvariationen gewonnen. Das Licht ist nun nicht mehr von abstrakter Kälte, es ist von brauner Tönung, in deren Wärme sich die Verbindung des Lichts mit der Materie ausdrückt. Freilich handelt es sich nicht um den Lichtduft der Venezianer, Ribera ist viel weniger atmosphärisch, das Licht verbindet sich bei ihm mit kompakter, erdhafter Materie.

Mit dieser Lichtbehandlung hängt die Führung des Pinselstrichs zusammen. Ribera verleugnet nicht, wie Caravaggio mit seiner polierten Oberfläche, den Pinselstrich. Dieser bleibt sichtbar. Die Malerei ist damit weniger illusionistisch, es wird dem Betrachter nicht verhehlt, daß er bemalte Leinwand vor sich hat. Ribera hat offensichtlich alla prima gemalt, feucht in feucht, in den früheren Werken mit verhältnismäßig trockener Farbe in borstigem Auftrag. Dann wird die Technik zusehends flüssiger, die hellen Werte sind weniger einzeln aufgesetzt als aus dem Ganzen herausgeholt. Schon diese pastose Malweise verhindert, daß das dargestellte Licht als von außen einfallendes erscheinen kann. Licht zeigt sich als helle Farbmaterie, also nicht in seiner abstrakten Qualität. Zudem ist durch die sichtbare Maltechnik die Illusion der Darstellung von Oberfläche von Dingen zerstört. Wie sehr sich Riberas Helldunkel von der realistischen Vorstellung eines Lichteinfalls lösen kann, zeigen einige Bilder der mittleren und späten Zeit, wo die Gestalten sich unter hellem Himmel befinden und trotzdem - durchaus nicht naturalistisch - mit Helldunkel gemalt sind. Das Licht ist nur dienstbar der Gestaltung der Materie. Ahnlich wie das Licht als solches verliert der Raum an Bedeutung, denn mit der fehlenden Illusion des Lichteinfalls fällt auch das damit verbundene raumschaffende Element weg.

Noch ein anderer Aspekt von Riberas Pinseltechnik ist hervorzuheben. Der Farbauftrag ist nicht nur reich an Abtönungen, er ist auch rauh. Das schafft zusätzlichen Reichtum, indem das Licht, welches das Gemälde beleuchtet (in der Kirche, im Museum), bei den vielen minimen Erhebungen seinerseits kleinste Licht- und Schatteneffekte schaff. Auch so verfängt sich Licht in der Oberfläche. Die jeweiligen Beleuchtungsverhältnisse sind also für die Erscheinungsweise entscheidend - das ist ein neuer Abtrag am zeitlosen Charakter des Gemäldes.

Auf die Farben selbst ist noch näher einzugehen. Es gibt bei Ribera kaum mehr Lokalfarben, fast nur noch Ưbergangstöne. Damit ist Caravaggios Antinomie zwischen Farbe und Licht (wie die Antinomie zwischen Materie und Licht) aufgelöst. Farbe stellt ein grundsätzliches Problem für die Helldunkelmalerei. (Man denke daran, wie um r 900 das Helldunkel im Namen der 


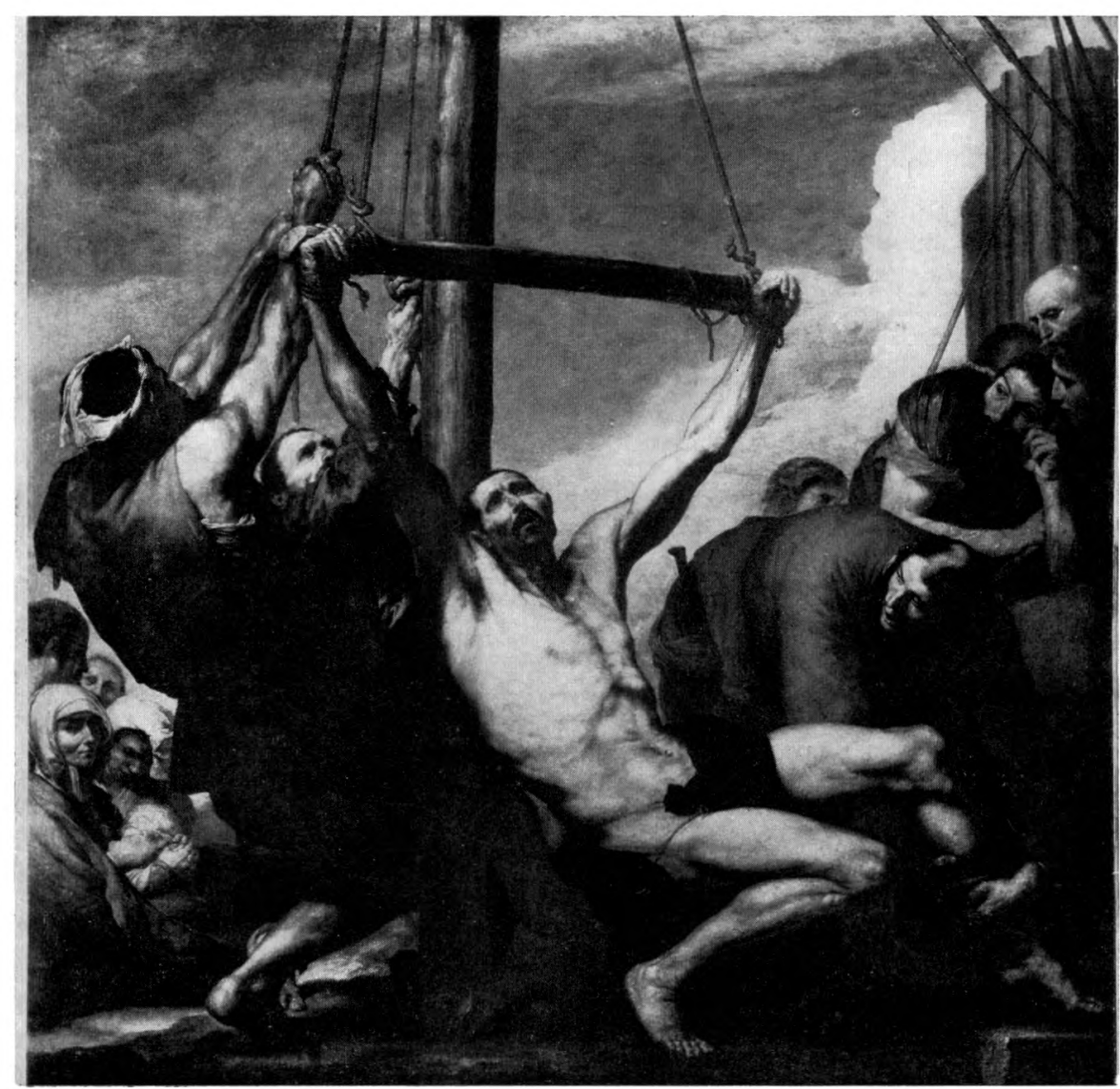

2. Ribera, Das Martyrium des heiligen Bartholomäus. Madrid, Prado

Farbe liquidiert wurde.) Im hellen Licht und im Dunkel des Schattens sind keine reinen Farben möglich. Bei Caravaggio gibt es noch intensive Farben, die aber vom Dunkel bedroht sind. Bei Ribera ist jeder Farbe das Dunkel schon beigemischt, Helldunkel und Farbe sind immer je schon vermittelt. Helligkeits- und Farbwert sind damit nicht mehr voneinander zu scheiden. Bei Caravaggio tragen die dunkeln Bildpartien deutlich den Charakter des Beschatteten. Ein dunkles Rot ist ein Rot, das wegen Mangel an Licht nicht rein in Erscheinung treten kann, ein defizientes Rot also. Bei Ribera ist eine dunkle Farbe ein eigener Wert, der durchaus für sich besteht, nicht eine Farbe, die an sich rein wäre, aber wegen des Dunkels nicht zu sich selber kommen kann. Das Dunkel ist bei Ribera damit nicht mehr einfach Negation. Ein Dunkelrot besitzt als positive Qualität die Dunkelheit (und ist nicht bloß ein Rot, das einer quantitativen Einschränkung seiner Qualität unterliegt). Selbst Schwarz ist nun eine Farbe. In bezug aufs ganze Bild heißt das, daß dieses nicht mehr ein Dunkelraum ist, in dem einzelnes durch das Licht in Erscheinung treten kann, Farbe sein darf. Das Bild ist bei Ribera durchwegs Farbe, da auch die dunkeln Partien Farbe sind. Bei dieser Vereinigung von Farbe und Licht ist zugleich Verwandtschaft und 
Unterschied zur venezianischen Malerei des 16. Jahrhunderts festzuhalten: auch dort verband sich Farbe mit Licht; doch diese Verbindung wurde ausgekostet als Spiel zwischen beiden Elementen, als schwebendes Gleichgewicht des nicht Entschiedenen, dem die Eindeutigkeit der Identität von Licht und Farbe fehlte.

Mit dieser Synthese von Licht und Farbe hängt die Bedeutung zusammen, die nun bei Ribera den Brauntönen zukommt. Noch nie vor ihm ist Braun derart dominant in der Malerei gewesen. Es ist keine reine Farbe, gehört weder zum Regenbogenspektrum noch zu den Komplementärfarben. Hergestellt werden kann es durch Mischung von Schwarz und Rot. Vor Ribera war es im wesentlichen Dunkelton, beschattete Farbe, bei ihm wird es zu einer selbständigen Farbe. (Die Ambivalenz des Brauns als Farbe und als bloßer Dunkelton spiegelt sich übrigens auch in der Sprache: das italienische Wort dafür, umbra, bedeutete ursprünglich Schatten, das Spanische besitzt noch kein Wort für mittleres Braun, sondern nur eines für Dunkelbraun, moreno, und das deutsche Wort braun bedeutet ursprünglich dunkel und entwickelte sich erst im Verlauf des I7. [!] Jahrhunderts zu einer Farbbezeichnung.)

Mit dem Ausschalten der Lokalfarbe verliert die Farbe bei Ribera den Charakter des Absoluten, es gibt keine reinen Werte mehr, alles ist tonige Abwandlung. Es stehen also nicht einzelne Farben nebeneinander, das Bild ist ein Heller- und Dunklerwerden ohne Werte, die einen ruhigen Fixpunkt abgäben. Die Farbigkeit ist selbst transitorisch, hat Zeitlichkeit in sich aufgenommen: sie ist ständige Abwandlung, und Abwandlung gibt es nur in der Zeit. So gewinnt Riberas Reichtum der Töne seine tiefere Bedeutung: unter dem Diktum der Zeitlichkeit interessiert nicht mehr das reine Sosein von Farben und ihr Zusammenklang, der ein statischer ist, sondern vielmehr die Abwandlung der Erscheinung, die ständige Verwandlung, das Übergehen vom einen zum andern. So ist Riberas Malerei nicht mehr Ausdruck der Bedrohung durch die Zeit, sondern selbst verzeitlichte Malerei, der eben gerade deshalb die Zeit nicht mehr fremdes Element ist. Dies läßt sich noch von anderer Seite her zeigen.

Bei Ribera läßt das Helldunkel die Körperlichkeit des Dargestellten ganz anders erscheinen als bei Caravaggio. Bei diesem wird die Körperlichkeit durch das scharf einfallende Licht gezeigt, bewußt gemacht. Das Dargestellte muß vom Betrachter wegen der Helldunkeleffekte in der Dreidimensionalität vorgestellt werden. In Riberas gemildertem Licht wirkt die Körperlichkeit weniger skulptural, dafür scheint sich das Plastischwerden gleichsam vor dem Auge des Betrachters zu vollziehen. Denn die Übergangstöne besitzen vermehrte Bedeutung, das Auge geht dem Heller- und Dunklerwerden nach, und auf dieses Werden kommt es an. Das Bild zeigt nicht in einem zur Dauer fixierten Augenblick dreidimensionale Körper, sondern schaff den Eindruck werdender Form. Man möchte geradezu in die Bilder hineingreifen und mitkneten. Der Schöpfungsprozeß ist präsent, wie ja auch der Pinselstrich bei Ribera sichtbar bleibt. Damit ist der Betrachter noch ganz anders als bei Caravaggio in einen zeitlichen Vorgang einbezogen. Wo Zeit herrscht, gibt es nichts Endgültiges: das Bild ist nicht mehr einfach vollendetes Werk, sondern teilt sich dem Bewußtsein mit als ein gewordenes, ja als ein werdendes. Wenn Caravaggio die Bedingtheit durch die Zeitlichkeit brüskierend gezeigt hat, so wertet Ribera gerade das aus, was durch die Zeit möglich ist: das Schöpferische als eigenen Wert. Der Akt des Gestaltens ist nun das Bedeutende, nicht ein Werk, das zeitentrückt aus dem Schöpfungsakt entsteht. In diesem zeitlichen Phänomen findet Riberas Kunst Sinn und Ziel.

So ist Riberas Malerei weniger schockierend als die Caravaggios, es geht nicht mehr um den Effekt einer Widersprüchlichkeit 3. Seine Malerei ist mit der Zeit versöhnt, ihr Abenteuer ist die Arbeit mit der Farbmaterie, deren schöpferische Abwandlung. 
Antonio Palomino, der spanische Kunstschriftsteller der Barockzeit, berichtet, im Kloster San Pablo in Sevilla sei ein Christus am Kreuz von Zurbaran so hinter einem Gitter im Halbschatten aufgehängt gewesen, daß die Leute gemeint hätten, es sei eine Skulptur. Die Anekdote kennzeichnet den Charakter von Zurbarans Helldunkeltechnik. Das Licht fällt illusionshaft ins Bild wie bei Caravaggio, so daß das Dargestellte deutlich und körperhaft in Erscheinung tritt. Es findet sich also nichts von Riberas pastoser Maltechnik, seiner Licht-Farbe-Vermischung. Kein Spiel von Glanzlichtern entfaltet sich, die Übergänge sind kontinuierlich. Bei aller Verwandtschaft mit Caravaggio ist auch der Gegensatz groß. Der Augenblickscharakter fehlt; Zurbaran zieht ruhige Motive vor, und selbst wo Handlung herrscht, ist kaum Bewegung zu spüren. Das Licht zeigt als klar gebündeltes die Dinge als in sich ruhende und konzentriert den Blick aufs einzelne. Es sind nicht Details willkürlich herausgegriffen, vielmehr erscheinen die Gegenstände in ihrer Ganzheit, das Licht dient gerade ihrer deutlichen Erscheinung. Es gibt auch keine Atmosphäre, das Licht verfängt sich nicht in der Luft: ohne Trübung sollen die Dinge erscheinen, das Drumherum ist nicht wichtig; das Licht hebt das Wesentliche heraus und läßt Unwesentliches im Dunkeln. Selbst lebende Menschen scheinen in die Dauer der Skulptur erhoben und wirken in dieser Abwesenheit von Bewegung, von Leben als Dinge. Das Fließend-Werdende von Ribera, die zeitliche Gespanntheit Caravaggios findet man nicht. Im kühlen, silbrigen Licht Zurbarans erscheint das Dargestellte in reinem, dauerndem Hiersein.

Damit stellt sich die Frage, ob das Problem der Zeitlichkeit in Zurbarans Helldunkelmalerei überhaupt vorhanden sei. Es ist dies durchaus der Fall: denn gerade daß die Bilder Zurbarans ausgesprochen ruhig wirken, zeigt ihre temporale Qualität. Ruhe ist Bewegungslosigkeit angesichts der Möglichkeit von Bewegung, ist Bleiben in der Zeit. In hochmittelalterlicher Malerei ist die Absenz der Bewegung nicht als solche spürbar, die Bilder sind bewegungslos, aber drücken nicht Bewegungslosigkeit aus. Bei Zurbaran ist Dauer ausgedrückt als ein Nicht-Berührtsein von zeitlicher Veränderung innerhalb der Zeit. Dauer setzt als Negation von Zeitlichkeit diese gerade als vorgängige Erfahrung voraus.

Daß das Dargestellte auf Zurbarans Gemälden in seiner ruhenden Dinghaftigkeit erscheint, ist nun tiefer zu fassen. Wenn Vergänglichkeit als in der Diesseitigkeit bestimmend erfahren ist, so entsteht das Staunen darüber, daß es doch Dinge gibt, daß etwas da, unwiderruflich da ist. Angesichts der Vergänglichkeit wird das bloße Sein der Dinge zum Rätsel. Was ist ein Dasein, das nicht ein Immersein ist? Was ist ein Seiendes vor dem Hintergrund seiner Vernichtung, der Zeit? Das Staunen über die bloße Tatsache des Daseins von Dingen, das Rätseln um ihr Sein drückt sich bei Zurbaran aus. Darum zeigt er die Dinge so deutlich im relativierenden Licht. Es handelt sich gleichsam um einen Caravaggismus mit umgekehrtem Vorzeichen: Caravaggio zeigt die Relativität der vorschnell angenommenen Zuverlässigkeit des erscheinenden Seins, Zurbaran staunt darüber, daß trotz dieser Relativität eben doch Dasein ist.

Von daher erklärt sich, daß, bei Zurbaran gerade Alltägliches, Einfaches Thema wird und eine besondere Weihe erhält. Seine Stilleben wirken mit ihrem Wesentlichwerden der einfachen Gegenstände fast religiös. Es ist Heiligkeit der Alltäglichkeit, wie Zurbaran sie gefunden hat in den Klöstern, für die er immer wieder gemalt hat, bei den Mönchen, die immer wieder Bildthema bei ihm sind. Zurbarans Gemälde tragen selbst mönchischen Charakter, sind in der Zeit transzendierte Zeit 4 . Wenn Zurbaran ein Thema wie die Herkulesgeschichte gestaltet, wo Kraft und Tat zum Ausdruck kommen sollten, entstehen eher peinliche Gebilde. 


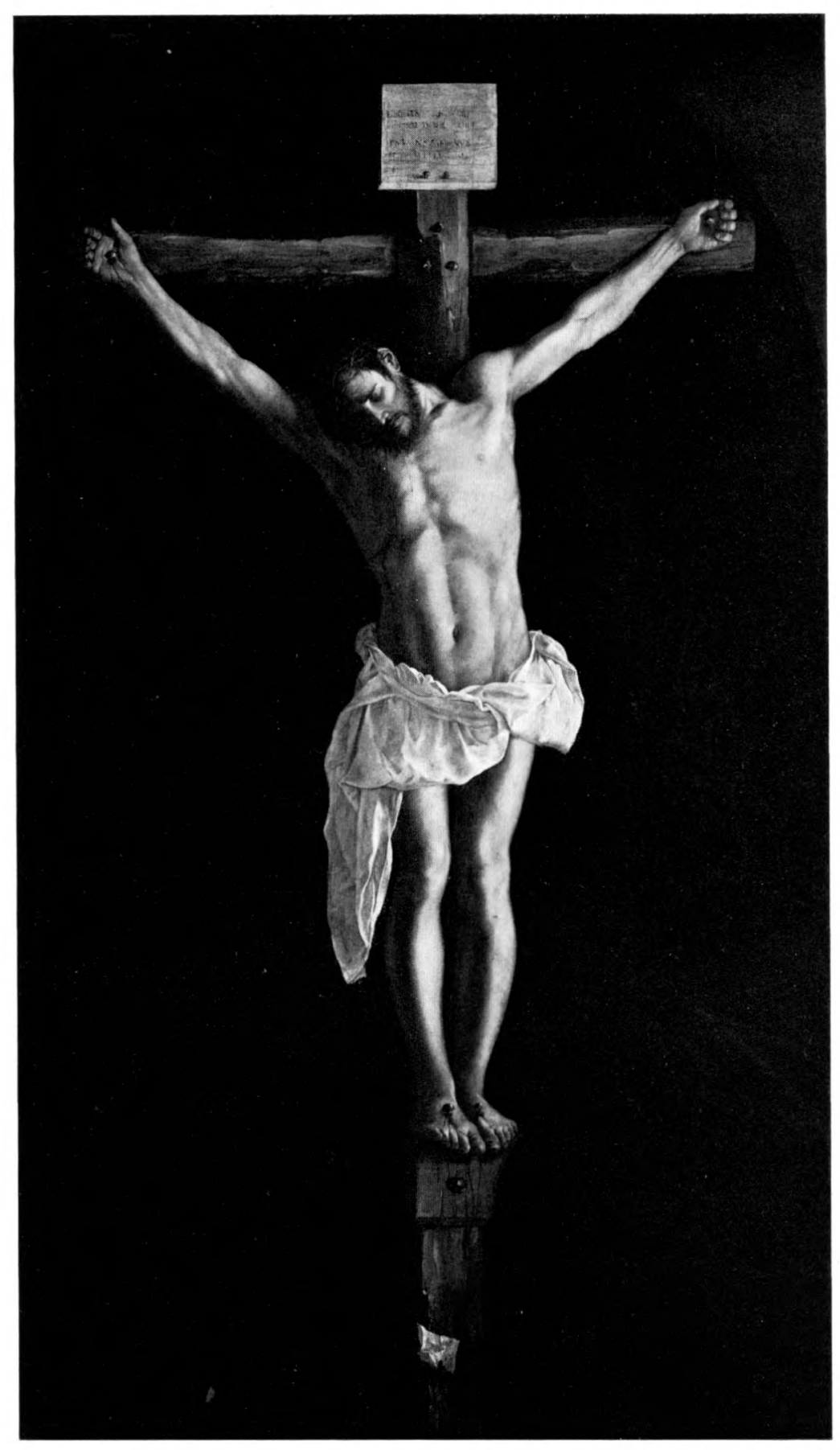

3. Zurbaran, Christus am Kreuz. Chicago, Art Institute 
Das Paradox von Dasein in vernichtender Zeitlichkeit wird besonders deutlich im immer wiederkehrenden Todesmotiv bei Zurbaran. Der Tod ist nicht als Geschehen gezeigt, vielmehr erscheint der tote Körper: Tod, Vernichtung ist thematisch, und doch ist Gegenständliches, der tote Körper, dargestellt. Dieses Sein des Nichtseins schaff eine seltsame Faszination. Todeserfahrung ist Hintergrund für das Rätseln um das Dasein, das Sein der Dinge ist auf Zurbarans Bildern Dasein in bezug auf den Tod. Vor dem dunkeln Hintergrund sind die Dinge bei Zurbaran oft wie in einen unendlichen Raum gestellt. Indem das Licht von unbestimmtem Ort her in den dunkeln Bildraum einbricht, wirken weder Zeitlichkeit bestimmt noch der Raum begrenzt. Kein größeres Ganzes, keine umfassende Totalität ist gegeben in solcher Unbestimmtheit, bloß das einzelne in seiner Tatsächlichkeit ist da. Hinter dem Dasein ist kein allgemeines Sein verbürgt, das Besondere trägt in sich selbst die Wesentlichkeit, es ist selbst sein Wesen. Das ist der Grund für den Eindruck der Weihe, den die gemalten Dinge bei Zurbaran vermitteln. Zurbarans Gemälde werden so in besonderem Maße zu Andachtsbildern: Versenkung in ein einzelnes sind sie, und das einzelne ist Wunder. Es ist ruhend, dauernd - Bewegtes wäre zu flüchtig, im Ruhen ist Vergänglichkeit transzendiert. In der Andacht, der Konzentration auf das Gemalte, das durch seine intensive Beleuchtung den Blick an sich hält, ist der Betrachter als lebender Mensch zur Ruhe gebracht für die Dauer des Anschauens. Auch in Zurbarans Malerei ist der Betrachter so hineingenommen in die Temporalität des Bildes. Diese Temporalität ist freilich nicht das Schöpferische von Ribera, die Dauer des Bildes entspricht gerade nicht der zeitlichen Bedingtheit des menschlichen Lebens; deshalb haftet der Wirkung von Zurbarans Malerei auf den Betrachter immer der Charakter der Fremdheit an.

\section{Georges de La Tour}

In Georges de La Tours Bildern herrscht eine ähnliche Ruhe und Stille wie bei Zurbaran. Doch ist das Licht bei ihm nicht silbrig kühl, sondern warm und rötlich. Das gibt den Bildern einen intimeren Charakter. Sie sind nicht stillebenhaft im Sinne einer nature "morte“, sondern sind wirkliches stilles Leben. Licht ist hier zugleich Wärme. Besonders bedeutsam ist dabei, daß das Licht nicht von einem unbekannten Außen hereinfällt, sondern in den meisten Bildern die Lichtquelle selbst dargestellt ist (als Kerze, als Fackel). Damit dient das Licht nicht bloß der genauen Darstellung der Gegenstände wie bei Zurbaran, sondern ist selbst Gegenstand der Darstellung. Es fällt nicht als abstrakt Unbekanntes ein, sondern ist erklärt, zeigt sich in seiner eigenen Bedingtheit - ist also nicht mehr selbst nur das Bedingende. Es handelt sich gleichsam um ein materialistisches Lichtverständnis, das Licht ist irdisches Licht. So ist es nicht mehr absolute Kategorie, sondern ist selbst der zeitlichen Bedingtheit der Erscheinungswelt unterworfen. Innerhalb ihrer Bedingtheit erhellt sich diese selbst. Die Bilder sind somit ganz in sich geschlossen, in sich ruhend. Sie ziehen damit den Betrachter in ihre eigene Welt wie in ein Refugium: das macht ihren besonderen Andachtscharakter aus. Im umfassenden Dunkel (kein Licht kommt ja von außen herein) ist gleichsam eine Stelle gezeigt, wo sich Dasein realisiert und durch das eigene Licht selbst verbürgt. Das Bild ist abgerückt von der übrigen Welt, es ist gleichsam eine Zelle Leben, weniger dinghaft als bei Zurbarans Konzentration auf die Gegenständlichkeit. Dargestellt sind kleine, stille, einfache, undramatische Szenen. Angesichts der Vergänglichkeit ist das Große ja nicht bedeutender als das Kleine. Große Gestik, Idealisierung des Dargestellten und Reichtum würden gerade ablenken von dem einfachen Wunder der Tatsache bloßen Daseins. Wie an einem Ort die verrinnende Zeit gefüllt ist mit dem Leben einer kleinen Szene, das zeigt 


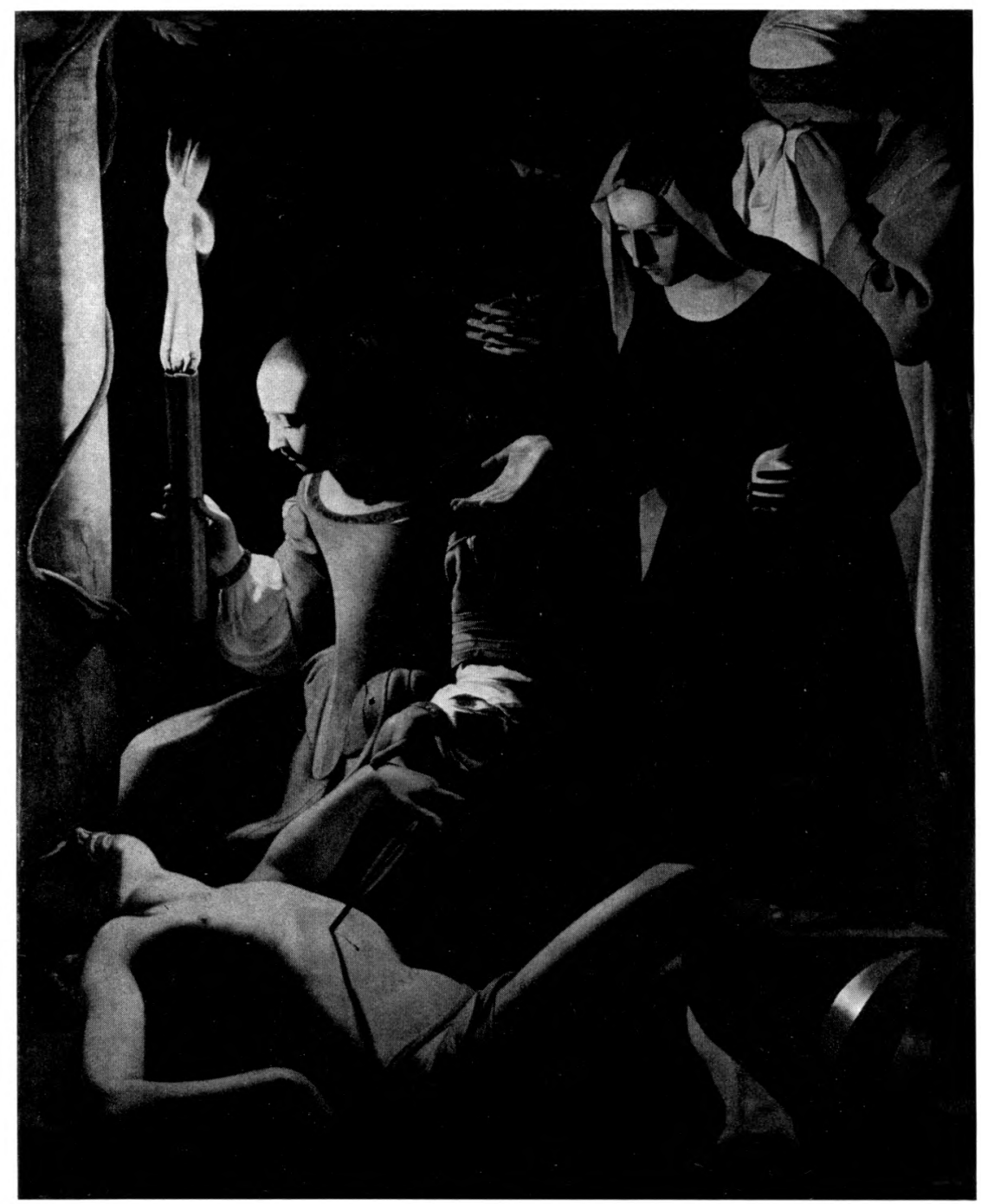

4. G. de La Tour, Sebastian, von der heiligen Irene und ibren Frauen betrauert. Berlin, Statliche Museen

G. de La Tour. Nur als so gefüllte erscheint bei ihm Zeit, als realisierte, nicht als abstrakte. In der beruhigenden Dauer dieser Bilder ist Zeit aufgehoben im mehrfachen Wortsinn: erhalten und zugleich überwunden, als Dauer realisiert. Die Szenen sind für sich da, durch Zeit vermittelt, aber nicht unter fremdem Gesetz. -

Durch die ruhige Simplizität unterscheidet sich G. de La Tour von der übrigen Genremalerei seiner Zeit. Es geht ihm nicht um amüsante Darstellungen des Alltagslebens, um Anekdotisches. Es geht um die Tatsache des Lebens in aller Verlorenheit in der Dunkelheit. Solcher Gehalt führt gerade zu einem Abstrahieren von besonderen Details, so daß G. de La Tours Bilder oft sehr abstrakt wirken. Nicht das Konkrete an sich, sondern die Tatsache der Konkretheit, ihr Dasein als stilles Für-sich-Sein ist Gehalt der Bilder. 


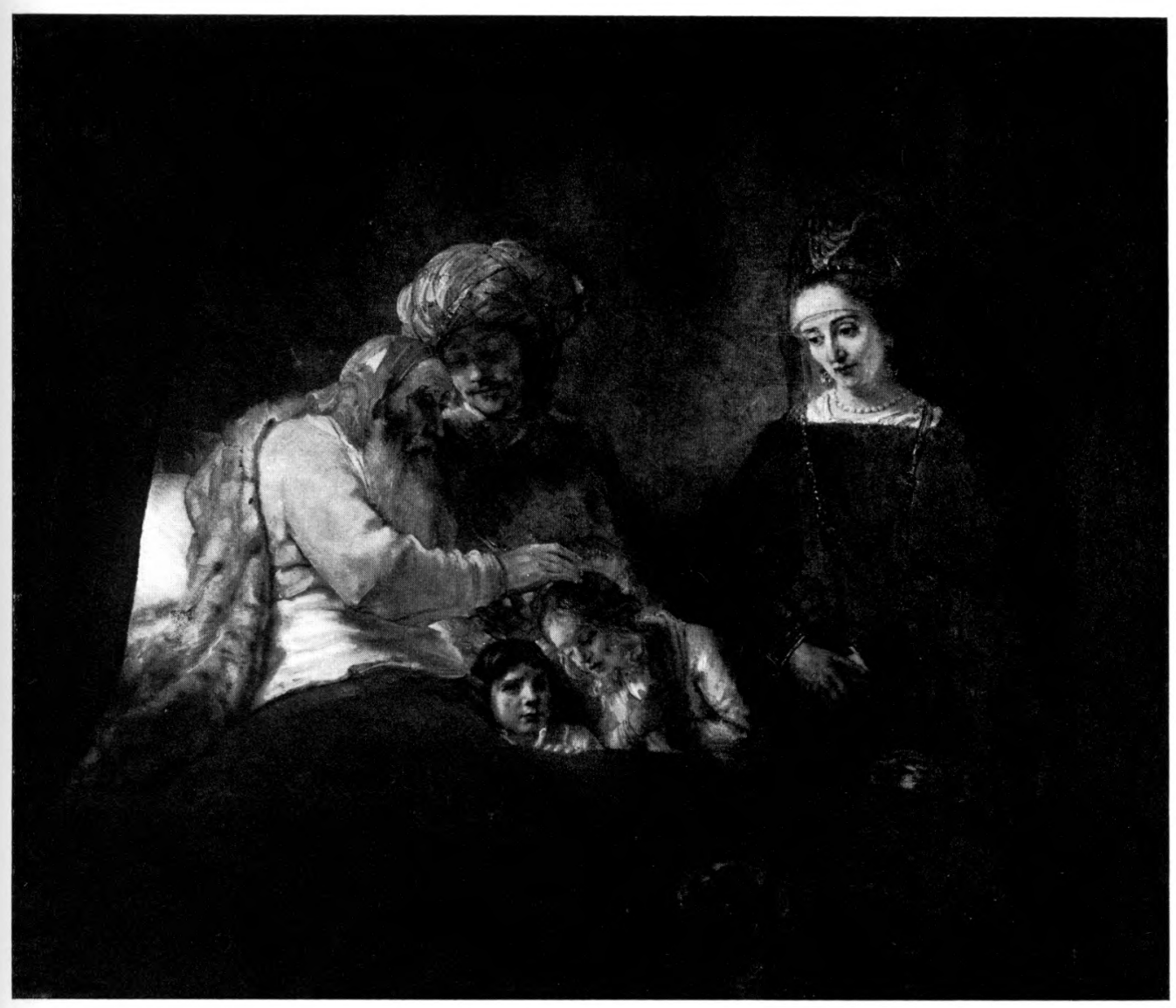

5. Rembrandt, Jakobs Segen. Kassel

Re m brandt

Rembrandt ist in seiner malerischen Technik Ribera verwandt; was von dessen Sichtbarlassen des Pinselstrichs gesagt wurde, gilt auch für Rembrandt. Der Unterschied zwischen beiden zeigt sich in erster Linie im Atmosphärischen von Rembrandts Malerei. Es gibt in ihr keine derart dichte Materialität wie bei Ribera. Das Licht verfängt sich schon in der Luft, und die Gegenstände scheinen in diese Lichtluft eingetaucht. Materie, Licht und Luft sind nicht scharf zu trennen als verschiedene Elemente. Man weiß nicht, fällt das Licht auf die Oberfläche der Dinge, die es reflektieren, oder leuchten die einzelnen Materienteilchen selbst. Das Licht pflanzt sich fort von einer Quelle kommend und zugleich sich immer wieder neu entzündend in der Verbindung mit der Materie. Das Einzelne verliert in solcher Vermischung sein Eigengewicht, eins geht ins andere übers. Es entsteht eine Rembrandt ganz eigene Raumhaftigkeit, die sich in der Vorliebe für Interieurs motivisch ausdrückt. Der Raum entsteht nicht durch eine äußere Begrenzung, sondern durch das Malen seines Inhalts, der erleuchteten Luft, in die die Dinge ein- 
getaucht sind. Die Begrenzung selbst bleibt unbestimmt. Das Gegenständliche erscheint wie eine Verdichtung dieses atmosphärisch gefüllten Raums. Von Oberfläche kann nicht mehr die Rede sein, so sehr ist sie durch den Pinselstrich aufgelöst. Nichts Festes und Festumrissenes existiert mehr, alles scheint sich aus der Einheit herauszuentwickeln, ein ständiges Werden. Die Unbestimmtheit macht es dem Betrachter unmöglich, genau zu erkennen; die Grenzen zum bloß Vermuteten bleiben offen, beim Sehen entdeckt und ahnt er immer Neues: so überträgt sich der Charakter des Werdenden unmittelbar auf den Betrachter selbst. Zeit ist damit selbst im Bild enthalten als Werden, als Wandlung der Materie, als Leben. (Zu diesem Beinhalten von Zeit vergleiche man die motivische Vorliebe Rembrandts für charaktervolle, schicksalgezeichnete und alte Gesichter, wo auch, etwa im Gegensatz zu Caravaggios Jünglingen, die durchlebte Zeit präsent ist.) Wenn bei Ribera das Bild unmittelbar Ausdruck des schöpferischen Aktes war, so scheint bei Rembrandt das Bild selbst zu leben. Es ist mehr als bloß Objekt des Gestaltenden.

Dunkelheit gewinnt so bei Rembrandt eine neue Qualität. Aus ihr heraus scheint das Sichtbare zu kommen, aber nicht so wie bei Caravaggio, wo das Licht grell einzelnes beleuchtet und der Finsternis entreißt. Durch die fließende Grenze zwischen Dunkel und Sichtbarkeit entwickelt sich die Erscheinungswelt aus dem unbestimmten Grund heraus, der als tragender Muttergrund wirkt, aus dem heraus die sichtbaren Gegenstände geboren werden. In sehr genauem Wortsinn kann hier von Erscheinung gesprochen werden: das Sichtbare scheint, leuchtet aus dem Dunkel heraus. Sein Sein ist Schein - auch in dem Sinn, daß dieses Sein sich nicht selbst verbürgt, sondern scheinhaft ist und nur in Verbindung mit dem Ganzen sein Wesen hat. Da im Dunkeln, das bei Rembrandt nie reines Schwarz ist, Gegenstände noch halb sichtbar oder bloß erahnbar sind, wird dieses zu etwas Geheimnisvollem, das birgt und verbirgt. Dem Verborgenen scheint ebenso große Bedeutung zuzukommen wie dem deutlich Erkennbaren. Wenn Rembrandt zum Beispiel gern in Porträts die Augen beschattet wiedergibt, so verbirgt er gerade das, was besonders stark einem Gesicht Ausdruck gibt; gerade im Blick glaubt man mehr als bloße äußere Erscheinung der Person zu fassen: es wird also das, was Außerlichkeit übersteigt, in geheimnisvollem Dunkel gelassen, um wirklich den Raum für dieses Mehr offenzulassen. In solcher Weise läßt das Dunkel bei Rembrandt immer ein Mehr als bloße Sichtbarkeit und Materialität erahnen. Man hat in bezug auf Rembrandt immer wieder von Vergeistigung, mystischer Vertiefung, Beseelung gesprochen: mit solchen Worten versucht die Sprache die Rembrandtsche Transzendierung der Materialität zu fassen. Im Grunde genommen kann aber dieses Mehr gerade nicht benannt werden, es ist wirklich nur ein Erahntes, Rembrandts Kunst ist Bewegung auf etwas hin, das sich der Erfassung entzieht. Das Geheimnis ist ihr eigen. In diesem Auf-etwas-hin zeigt sich die Temporalität von Rembrandts Kunst: nicht als Sein, sondern als Bewegung existiert sie, alles Statische, das sich der Zeit entzöge, fehlt ihr. Dem Betrachter wird nichts Festes und Fertiges gegeben, bei dem er sich beruhigen könnte. Er wird im Bild und durch das Bild weitergetragen. Insofern geht Rembrandt gerade den umgekehrten Weg als Zurbaran, der das statische Sein zum Ausdruck brachte. Rembrandts Gemälde sind durchwegs Übergang, von dunkel zu hell, von sichtbar zu unsichtbar, von einer Form zur andern, von einer Farbe zur andern.

So ist bei Rembrandt das Helldunkel das Prinzip des Übergänglichen (im äußersten Gegensatz zur Malerei mit Lokalfarben, die gerade vom Sosein des Einzelnen ausgeht). Wie sehr Helldunkel reines Prinzip der Darstellung werden kann, zeigt die Tatsache, daß die realistische Erklärung des Lichts oft unmöglich ist: es ist nicht zu entscheiden, ob Tag-oder Nachtbeleuchtung herrscht und woher das Licht kommt. Beim frühen Rembrandt gibt es noch deutlich die 
realistische Motivierung, of mit anekdotischem Einschlag, aber zusehends verliert sich dieser eindeutige Beleuchtungscharakter, was mit einer Vermischung der Helldunkel- und der Farbkategorie zusammengeht. Das Licht materialisiert sich, die Stofflichkeit entmaterialisiert sich: Zeichen dafür, daß irdisches Leben gerade durch die Vermischung der reinen abstrakten Kategorien entsteht.

Im Vergleich zu solcher Malerei ist Ribera geheimnislos. Er arbeitet als selbstherrlich Gestaltender mit der Farbmaterie, die nicht transzendiert wird. Seinem erdigen Braun fehlt der spezifisch Rembrandtsche Goldglanz, der das Dunkel in sich zu bergen scheint und zugleich wie höheres Leuchten wirkt. Dies ist immer wieder als religiöse Transzendenz erklärt worden. Es gibt bei Rembrandt jedoch kein verbürgtes Jenseitiges, Ideales. Er hat mit Caravaggio die Wendung zum Irdisch-Realen mitvollzogen. Aber dieses erweist sich ihm als das nicht Bestimmbare, seine Bedingtheit macht aus, daß man an kein Ende kommt: immer bleibt noch Geheimnis stehen. Die Bilder sind ein Nachsinnen, Nachgrübeln. Die Zeitlichkeit ist Bedingung für das Geheimnis, für die Tatsache, daß ein Letztes, Uberzeitliches nicht faßbar, höchstens erahnbar ist. Darum hat Rembrandts Kunst den Abbildcharakter abgelegt. Was erscheint, ist nur Teil eines größeren Ganzen, das sich nicht zeigt. Im ständigen Wandel erweist sich diese Relativität der Erscheinungswelt. Rembrandts Malerei will sich nicht mit ihrem bloßen vorläufigen Sosein begnügen, kann aber das Ganze auch nicht fassen, weil dieses dem Menschen entzogen ist. In dieser Malerei zeigt sich, wie der Mensch keine Souveränität besitzt und auch nicht in mittelalterlicher Weise in einen festen göttlichen Ordo eingefügt ist.

Mehr als bei anderen Malern wandelt sich das Helldunkel bei Rembrandt auch innerhalb des Schaffens. In frühen Werken mit forcierten Lichteffekten und starken Glanzlichtern wird das Augenblickliche noch betont. Das Phänomen Zeit wird pointiert als Plötzlichkeit zum Ausdruck gebracht. Die Nähe zum Caravaggismus ist deutlich. Im Gegensatz zu seinen holländischen Zeitgenossen zeigt sich schon da Rembrandts Interesse für zeitliches Geschehen. Erst in den reiferen Werken entsteht das fließende Heller- und Dunklerwerden der Leinwand, das Eindringen des Lichts in die Materialität, wo Zeit nicht mehr durch zugespitzten Effekt, sondern durch ständige Wandlung zum Ausdruck kommt. Die Helldunkelgegensätze können damit wieder geringer werden, und Farbe kann wieder größere Bedeutung erlangen.

\section{Zur wissenschaftichen Literatur}

Die Literatur zum Helldunkel ist im Hinblick auf die Bedeutsamkeit des Problems nicht groß. In Werken über die Barockmalerei und in Monographien zu den einzelnen hier behandelten Künstlern wird zwar über das Phänomen gesprochen, und manche Bemerkung ist auch für die hier dargelegten Gedanken anregend gewesen, aber sehr weit wird die Analyse jeweils nicht getrieben. Einige interessante Ansätze zu einer Vertiefung des Problems finden sich im Kapitel „Rembrandts malerische Ansicht und Weltansicht" in der freilich schon recht alten Rembrandt-Monographie Carl Neumanns (Berlin/Stuttgart I902, S. I 54 ff.). Bedeutsam ist immer noch - gerade für den Versuch, eine positivistische Betrachtungsweise in Richtung des Philosophischen zu überschreiten - Georg Simmels Buch über Rembrandt - ein bis jetzt nicht wiederholtes Unternehmen („Rembrandt; Ein kunstphilosophischer Versuch“, Leipzig 21919).

Das eigentliche grundlegende Werk für die Betrachtung des Helldunkels ist Wolfgang Schönes umfassendes Werk "Uber das Licht in der Malerei“ (Berlin 1954). Zu den einzelnen hier behandelten Künstlern bietet das Werk natürlich angesichts seines weit gespannten Rahmens 
nicht viel. Seine grundsätzlichen Darlegungen zur Lichtproblematik sind aber entscheidend auch für das hier Ausgeführte gewesen. Das Werk hat überhaupt mannigfache Anstöße gegeben; besonders im Seminar von Hans Sedlmayr in München wurde eine interessante Diskussion ausgelöst: einige Aufsätze in den kunsthistorischen Berichten des Seminars geben einen Einblidk (Hefte des kunsthistorischen Seminars der Universität München, herausgegeben von Hans Sedlmayr, besonders Heft s mit dem Gesamttitel „Uber Farbe, Licht und Dunkel“, 1959, ferner der Aufsatz von Wilhelm Messerer "Die Zeit bei Caravaggio" im Heft 9-10, 1964, S. 55 ff.). Fruchtbar für eine eingehende Betrachtung der Helldunkelproblematik ist immer noch Theodor Hetzers Buch über „Tizian, Geschichte seiner Farbe“ (Frankfurt a. M. ${ }^{2}$ I 948).

Am nächsten dem hier vorgelegten Versuch steht freilich ein Aufsatz, der zunächst fern zu stehen scheint. Es handelt sich um Halldor Soehner, „Velazquez und Italien“, in der Zeitschrift für Kunstgeschichte $18 / 1955$, S. I ff. Soehner analysiert unter anderem das Helldunkel des jungen Velazquez im Vergleich zu Caravaggio und geht gerade auch auf eine temporale Interpretation ein. - Zum Teil beeinflußt davon ist Emil Maurers kurze Betrachtung von Caravaggios Helldunkel in der Festschrift Hahnloser (Basel/Stuttgart I96 I, S. 393 ff.), eine Interpretation, die der hier versuchten ebenfalls nahesteht.

Nicht zu vergessen ist, daß vieles, was in dieser Untersuchung ausgeführt wurde, auf Anregungen zurückgeht, die sich in Heinrich Wölfflins Grundbegriffen finden („Kunstgeschichtliche Grundbegriffe", Basel/Stuttgart ${ }^{13}{ }_{1963}$, besonders der Begriff des Malerischen).

Ein Versuch, mit philosophischen Kategorien an die Kunst heranzugehen, findet man neuerdings in Wolfgang Zuckers Aufsatz "In the Light of Being“ im Journal of Aesthetics and Art criticism, I968, S. I $53 \mathrm{ff}$. Hier wird die Lichtbehandlung auf eine ontologische Aussage hin untersucht (im Gefolge der Philosophie Martin Heideggers), ohne daß allerdings auf das Problem der Zeitlichkeit eingegangen würde. - Zum Problem der Zeit in der bildenden Kunst andererseits findet man einiges in Dagobert Freys "Gotik und Renaissance als Grundlagen der modernen Weltanschauung“, im Kapitel "Das Zeitproblem in der bildenden Kunst" (Augsburg 1929). Die Problematik wird freilich nicht aus dem Phänomen des Helldunkels heraus entwickelt. Fast ganz nur von ikonographischer Seite geht Erwin Panofsky in seinen "Reflections on Time“ in den "Problems in Titian“, New York 1969, aus.

Für die vorliegende Arbeit sind außer der kunstgeschichtlichen Literatur noch andere Untersuchungen anregend gewesen. So ist Heideggers Werk "Sein und Zeit“, Tübingen 7I953, selbstverständlich für solche temporale Deutung wichtig, dazu auch die Auswertung dieses Denkens für die literarhistorische Interpretation durch Emil Staiger (vgl. bes. „Die Zeit als Einbildungskraft des Dichters", Zürich ${ }^{3}{ }_{96}$ ). Zu nennen ist ferner Georges Poulets temporale Interpretation der Geistesgeschichte in seinen „Etudes sur le temps humain“, Meaux 1965. In der philosophischen Begrifflichkeit ist diese Arbeit zudem stark der Hegelschen dialektischen Logik verpflichtet (vgl. besonders G. W. F. Hegel, "Wissenschaft der Logik“, in verschiedenen Ausgaben).

\section{Zur Methode}

Die obigen Ausführungen beschreiben nicht Kunstwerke, sondern lesen aus ihnen bestimmte Haltungen zu menschlichen Problemen heraus. Der Gehalt ist nicht in Schönheit, Tiefe oder Eindrüdklichkeit gesucht worden (wie das in popularisierender Kunstgeschichtsschreibung leider viel zu häufig geschieht), sondern in einem differenzierteren "message“, das je bei den verschiedenen Künstlern verschieden ist. Als Ausgangspunkt ist etwas Maltechnisches gewählt 
worden, das Helldunkel, was im Gegensatz etwa zu einer motivischen Untersuchung eher vor einer Literarisierung des Kunstwerks bewahrt. Damit, daß ein einzelner Aspekt herausgegriffen wurde, kam natürlich jeweils nur eine bestimmte Seite des betreffenden Künstlers zum Vorschein; - eine willkürliche Einschränkung bildet ferner die Tatsache, daß auf Unterschiede innerhalb des Werkes eines Künstlers fast gar nicht eingegangen wurde. Es ist damit nicht gemeint, daß diese Unterschiede nicht wesentlich seien.

Bei einer philosophischen Interpretation wie der hier gegebenen drängt sich das Problem der Sprache auf. Denn das, was gesagt werden kann, hängt von der heute vorhandenen Sprache ab: die Entwicklung der Begrifflichkeit, wie sie vom Denken in geschichtlichem Prozeß zunehmend geleistet worden ist, bestimmt die Art der Ausführungen. Grundsätzlich gesehen ist ein Sprechen über das Helldunkel von vorneherein eine Transponierung in ein anderes Medium: was malerisch da ist, wird zu etwas Begrifflichem. Das ist ein willkürlicher Akt, dem Gemälde selbst fremd. Was ausgeführt wurde, ist also nicht in einfacher Weise objektiv die Problematik des Helldunkels. Es ist die Begegnung des Denkens mit dem Kunstwerk, wobei das Kunstwerk zwar Kriterium für das Gesagte ist, aber nie mit dem Gesagten zusammenfällt (diese Zusammenhänge sind von der Hermeneutik schon lange grundsätzlich entwickelt worden, vgl. z. B. H.-G. Gadamer, „Wahrheit und Methode“, Tübingen ${ }^{2}$ I965, in der Kunstgeschichte wird das Problem freilich selten bewußt reflektiert). Wenn mich die Temporalität des Helldunkels interessiert, so ist das für das moderne Denken typisch: unsere Epoche hat eine besondere Affinität zu dieser Problematik. Schon darin also ist die Untersuchung historisch bestimmt. Wenn anderseits religiöses Vokabular (etwa bei der Betrachtung Rembrandts) stärker als gewöhnlich gemieden wurde, so geschah das in der Meinung, daß solche Sprache in der Kunstgeschichte heute allzu verbraucht sei und eher Barrieren für den Zugang errichte. Hier wurde eine andere Begrifflichkeit gewählt, - um von heute her die Aussage dieser Werke anzugehen.

A n merkungen

I Ausdruck von W. Schöne, Über das Licht in der Malerei, Berlin I954.

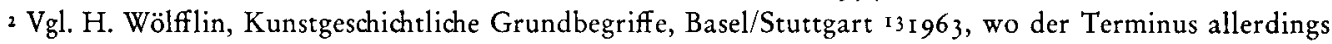
für die Charakterisierung der Barockmalerei gebraucht wird.

3 Ribera wurde seit seiner Zeit in dieser Hinsicht immer wieder falsch verstanden, man vergleiche etwa die Bemerkungen von Grautoff in N. Pevsner - O. Grautoff, Barockmalerei in den romanischen Ländern, Handbuch der Kunstwissenschaft, Wildpark-Potsdam I928, die allerhöchstens für den jungen Ribera und für Schulwerke zutreffen und am nüchternen Ernst von Ribera vorbeigehen. Vgl. die richtige Einschätzung von Elisabetk du Gué Trapier, Ribera, New York 1952, S. I, 3, 195. Die Vorliebe für Märtyrerszenen ist ein Suchen an den Grenzen des Menschseins: höchstens in solcher Negation des Irdischen gelingt Ribera Gestaltung von Außerirdischem, direktere Darstellung von Transzendentem war ihm weitgehend verwehrt.

4 Er ist, um mit Paul Guinard zu sprechen (Zurbaran, Paris 1960), zugleich peintre monastique und peintre monacal.

; Vgl. den Begriff des "Allverbindenden" in bezug auf Rembrandt bei H. Sedlmayr, Zugänge zu Rembrandt, in: H. S., Epochen und Werke II, S. 94 ff. Wien/Mündhen 1960. 